\title{
Compression-Induced Polycrystal-Glass Transition in Binary Crystals
}

\author{
Huijun Zhang ${ }^{1}$ and Yilong $\operatorname{Han}^{2,1, *}$ \\ ${ }^{1}$ Department of Physics, The Hong Kong University of Science and Technology, \\ Clear Water Bay, Hong Kong, China \\ ${ }^{2}$ The Hong Kong University of Science and Technology Shenzhen Research Institute, \\ Shenzhen 518057, China
}

(Received 10 January 2018; revised manuscript received 2 September 2018; published 9 November 2018)

\begin{abstract}
A polycrystal will transform into a glass when its grain size is sufficiently reduced. However, it is not clear how the polycrystal-glass boundary can be identified, whether the boundary is a sharp point or a crossover regime, and what features exist at this boundary. These questions have rarely been explored, partly because ultrafine-grained polycrystals are unstable. Here we compress binary single crystals composed of hard and soft disks into polycrystals and further into glasses by simulation. Through this approach, we can continuously increase the fraction of mismatched particles and produce tiny grains not accessible before. Such full spectrum of grain size enables us to study the polycrystal-glass transition. We observe rich structural, mechanical, dynamical, and thermodynamic features at the sharp polycrystal-glass boundary. These features could serve as criteria for the polycrystal-glass transition. Our analyses reveal the Hall-Petch and inverse Hall-Petch behaviors in two-dimensional polycrystals and identify three glass regimes. We find that compressing binary crystals can produce stable ultrafine grains only when compressible parts abound and appropriate two length scales exist in the soft-particle interactions. Our approach can guide the fabrication and stabilization of ultrafine-grained polycrystals. The observations cast light on the crossover regime of polycrystals and glasses.
\end{abstract}

DOI: 10.1103/PhysRevX.8.041023

Subject Areas: Materials Science, Soft Matter

\section{INTRODUCTION}

Solids come in two basic forms: crystal and glass. Glass is a noncrystalline amorphous solid with a disordered structure and mechanical rigidity whose relaxation time is much longer than the experimentally accessible time [1]. Most crystals are composed of many crystalline grains of varying sizes and orientations and are thus known as polycrystals. Fine-grained polycrystals have unique properties [2-4], but ultrafine-grained polycrystals are unstable and undergo grain coarsening constantly. Their fabrication is therefore a central problem in polycrystal studies [5]. When the mean grain size in a polycrystal is reduced to merely one or two particles, the solid would become completely disordered, as in a glass. A natural question to ask is below what grain size will the polycrystal become a glass, or in other words, where is the boundary between polycrystal and glass? Moreover, it is not clear what features might exist at the polycrystal-glass boundary

*yilong@ust.hk

Published by the American Physical Society under the terms of the Creative Commons Attribution 4.0 International license. Further distribution of this work must maintain attribution to the author(s) and the published article's title, journal citation, and DOI. and whether an ultrafine-grained polycrystal should be called a polycrystal or a glass, or if it is just a matter of terminology without basic importance. In fact, how to rigorously define polycrystal and glass remains an open question.

Glasses [1,6-10] and polycrystals [5,11-15] are often studied using different methods as two separate research fields. Their boundary is poorly studied because stable ultrafine-grained polycrystals are rarely available [5]. Achieving ultrafine-grained polycrystals would also enable exploring various novel properties in this regime. Specifically, understanding the transition between polycrystal and glass is crucial in fabricating ultrafine-grained polycrystals [5,12] and metallic glasses [8]. Crystalline order has been studied in colloidal [16-19], metallic [20], and silica [21] glasses, and glassy behaviors have been observed in crystals with high dopant [22] or defect [23] densities, or high polydispersities $[24,25]$. These studies, however, are neither about ultrafine-grained polycrystals nor about the polycrystal-glass boundary. Silicon [26,27] and water ice [28] crystals with anisotropic particle interactions have been compressed into amorphous structures. But because the compression occurred via a catastrophic collapse from a single crystal or large-grained polycrystal to a glass without experiencing an intermediate ultrafine-grained polycrystal state, these crystals cannot be 
used to explore the polycrystal-glass transition. Mixing different particles is an important way to fabricate metallic glasses [29] and polycrystals [5], but it is also not able to yield ultrafine-grained polycrystals extending into the glass regime. As a result, the polycrystal-glass boundary has rarely been explored.

Polycrystals are important in materials science and metallurgy. As the grain size decreases, grain boundaries will begin to dominate over conventional crystal behaviors. A variety of unique properties [2-4] emerge in such fine-grained polycrystals, but the large number of grain boundaries also render them more unstable. As a result, they are difficult to fabricate, and this is especially the case for ultrafine-grained polycrystals with a grain diameter $l<3 \mathrm{~nm}[5,13]$. Consequently, their properties are poorly understood.

Glassy dynamics is prevalent in disordered systems with rugged energy landscapes, but the nature of glass transitions remains a big mystery in science [1]. In the conventional glass transition, a supercooled liquid is rapidly quenched so that its disordered structure is frozen into an amorphous solid. Such a transition is difficult to understand because it involves a drastic slowing-down of dynamics but little structural change [1]. By contrast, the rarely studied polycrystal-to-glass transformation exhibits prominent structural change, which provides another angle for understanding the nature of glass formation.

In this work, we compress binary crystals to reduce the grain size continuously to the glass regime. We find a sharp polycrystal-glass transition with rich and robust features in both two dimensions and three dimensions. We mainly focus on the results in 2D systems because they are large enough to clearly show how material properties change with the grain size. Two-dimensional materials have been attracting increasing attention for their promising properties [30]. Important insights about 2D polycrystals [14,15] and glasses $[9,10,31-33]$ have recently been obtained in colloidal systems at the single-particle level. The trends of various material properties in the $2 \mathrm{D}$ polycrystal and glass regimes agree with the behaviors in typical 3D materials. Moreover, we show which parameter regime can produce stable 2D ultrafine-grained polycrystals near the polycrystal-glass boundary.

\section{COMPRESSING THE 2D BINARY CRYSTAL}

We perform molecular dynamics simulation of a mixture composed of 6400 hard disks and 6400 soft disks with (a)

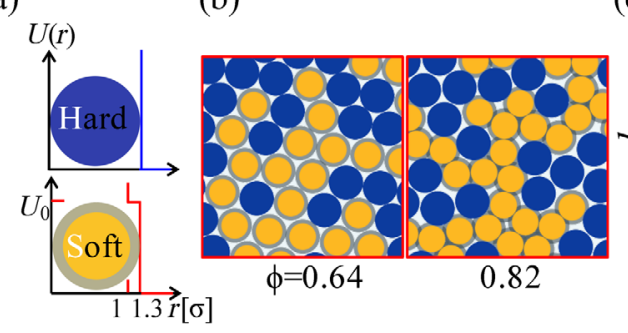

(c)

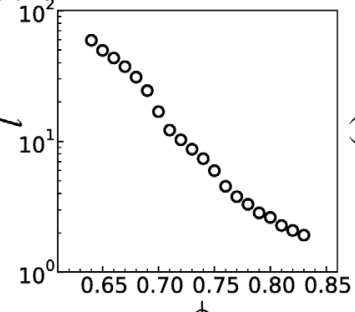

(d)

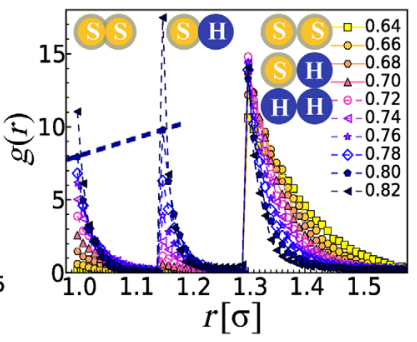

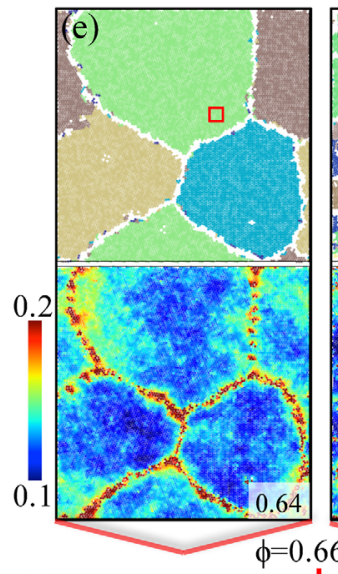
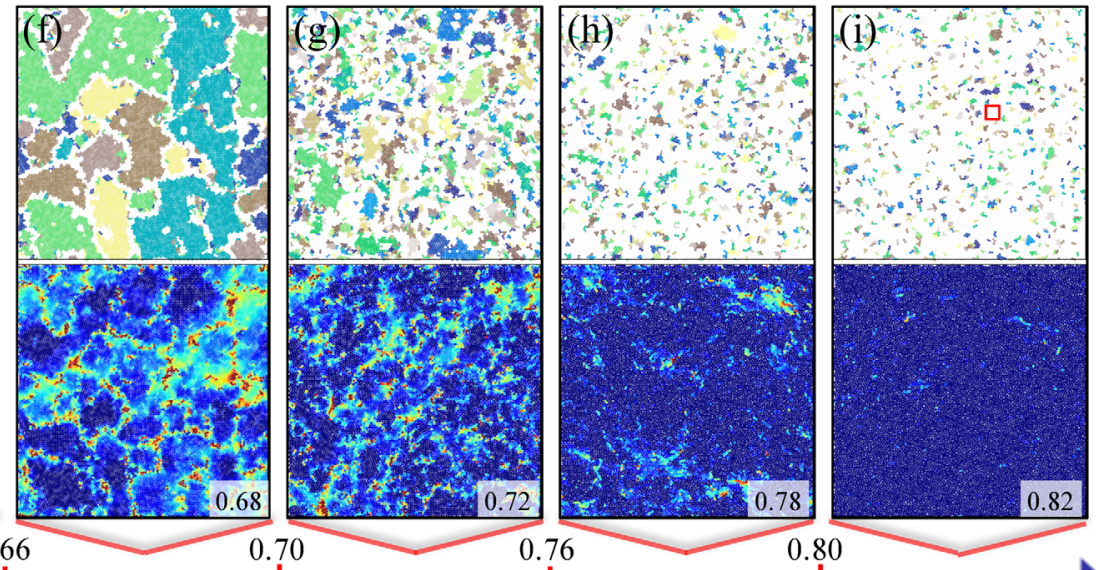

(j) Hall-Petch $:$ Inverse Hall-Petch

Glass

FIG. 1. The transformation of a binary polycrystal to a glass. (a) The interaction potentials of hard and soft disks. (b) Enlarged views of the regions marked by the small red boxes at $\phi=0.64$ in (e) and $\phi=0.82$ in (i). (c) Semilog plot of the weighted mean grain size $l(\phi)$. (d) The radial distribution functions $g(r)$ of the first-layer neighbors show three typical nearest-neighbor distances. (e)-(i) Structures (top) and dynamics (bottom) at $\phi=0.64,0.68,0.72,0.78$, and 0.82 , respectively, corresponding to the five regimes labeled in (j). Colors in the top panels show different grains and the white color marks the disordered particles. The bottom panels are colored according to the value of $L$ (see Appendix C). The initial single crystal at $\phi=0.62$ is shown in Fig. 11 in Appendix A. 
square-shoulder potentials [Fig. 1(a)]. Square-shoulder potentials have been widely used to describe metallic glasses based on cerium or cesium, water, granular, silica, and micelle systems [34-36]. We fix the temperature at $T=0.133 U_{0} / k_{B}$, where $U_{0}$ is the shoulder height [Fig. 1(a)] and $k_{B}$ is the Boltzmann constant. This temperature is well below the melting point and high enough to provide strong thermal motions. The phase behavior is solely determined by the area fraction $\phi$ or the corresponding pressure $P$. At low $\phi$, both types of disks have the same effective diameter of $1.3 \sigma$ and thus they form an "alloy" crystal [left-hand panel in Fig. 1(b)]. As the pressure (or $\phi$ ) increases, some of the soft disks are compressed, which distorts the local hexagonal structure and results in a glass [right-hand panel in Fig. 1(b)]. The quasistatic compression is explained in Appendix A.

\section{RESULTS}

\section{A. Structure and dynamics}

The local crystalline order of particle $j$ is characterized by the orientational order parameter $\psi_{6 j}$ (see Appendix B). It is used to distinguish crystalline grains from disordered particles [Figs. 1(e)-1(i) and Appendix B]. As $\phi$ increases, the grain size decreases continuously, which eventually leads to a glass [Figs. 1(c), 1(e)-1(i)]. The structure [Figs. 2(a)-2(c)], dynamics [Figs. 2(d) and 2(e)], and their correlation [Fig. 2(f)] all peak at $\phi=0.70$. Particles with strong dynamics, i.e., a large Lindemann parameter $L$ (see Appendix C), exist mainly at grain boundaries in polycrystals [Figs. 1(e) and 1(f)] and are randomly dispersed in glasses [Figs. 1(h) and 1(i)]. In Figs. 1(e)-1(i), disordered (white) particles in the top panels and high- $L$ particles in the bottom panels are most strongly correlated at $\phi=0.70$ [Fig. 2(f)]. The color distributions in Figs. 1(e)-1(i) show that near $\phi=0.70$, the structure and dynamics become most heterogeneous; i.e., their landscapes become most rugged. The ruggedness can be quantified by the fluctuations of structure and dynamics, e.g., the susceptibility of the orientational order $\chi_{6}$ (see Appendix B) and the relative standard deviation of $L, \operatorname{RSD}(L)$ (see Appendix C). The $\chi_{6}$ peak at $\phi=0.70$ [Fig. 2(a)] signals a crystal-glass transition. We find that this maximum fluctuation at $\phi=0.70$ corresponds to the peaks of $\operatorname{RSD}(L)$ [Fig. 2(d)], the variance of $\Delta L$ [Fig. 2(e)] (see Appendix C), the compressibility $\beta$ [Fig. 3(a)], the correlation between structure and dynamics [Fig. 2(f)] (see Appendix C), and the residual specific heat $c_{V}$ [Fig. 6(a)]; to the minima of the bulk modulus $K$ [Fig. 3(b)], the flow stress $\tau_{f}$ [Fig. 4(c)], and changes in the peak height and position of the density of state (DOS) [Fig. 5(d)]; and to the percolation of disordered particles [Fig. 2(c)]. These structural $\left(\chi_{6}\right.$ and percolation of disordered particles), dynamical $[\operatorname{RSD}(L)$ and $\operatorname{Var}(\Delta L)]$, mechanical $\left(\beta, K\right.$, and $\left.\tau_{f}\right)$, and thermodynamic ( $c_{V}$ and DOS) features consistently show that the boundary between polycrystal and glass lies at (a)

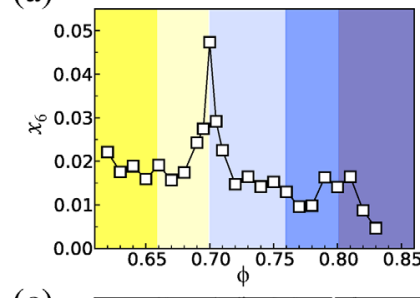

(b)
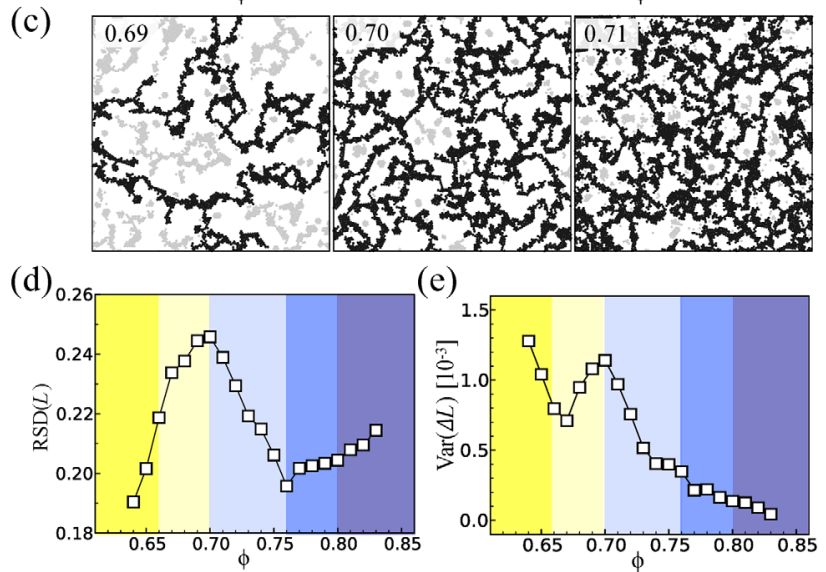

(e)

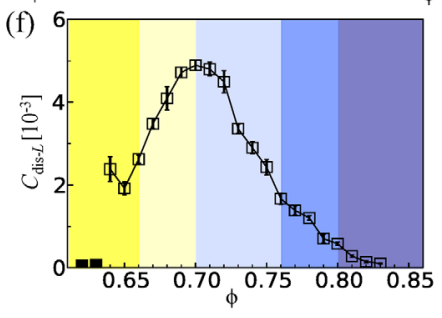

FIG. 2. (a) The structural fluctuation $\chi_{6}$. (b) The number of disordered clusters $N_{\text {dis-cluster }}$. (c) Morphologies of disordered clusters. Disordered particles are colored in black for the largest cluster and in gray for other clusters at $\phi=0.69,0.70$, and 0.71 . Black particles percolate at $\phi \geq 0.70$. Crystalline particles are not displayed. Dynamical fluctuations: (d) $\operatorname{RSD}(L)$ and (e) $\operatorname{Var}(\Delta L)$ (see Appendix C). (f) The correlation between dynamics and structural disorder (see Appendix C). The two solid square symbols at $\phi<0.64$ correspond to the single crystals whose disorder is nearly 0 .

$\phi=0.70$. These findings are remarkable because features at the polycrystal-glass boundary have rarely been revealed and the criteria for distinguishing a glass and an ultrafine-grained polycrystal are not clear. (a)

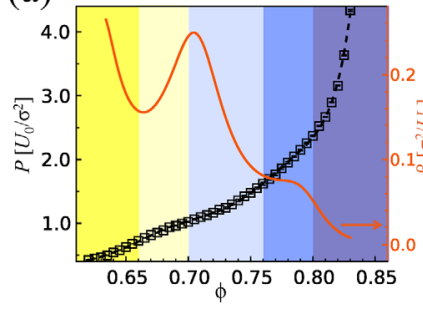

(b)

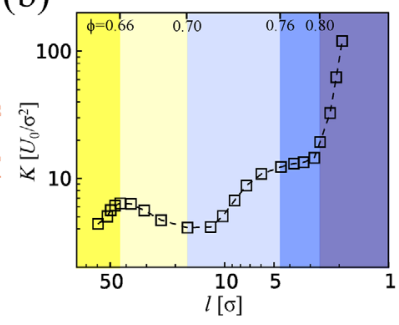

FIG. 3. (a) The equation of state $P(\phi)$ (squares) and the compressibility $\beta$ (curve). (b) Bulk modulus $K(l)$. 

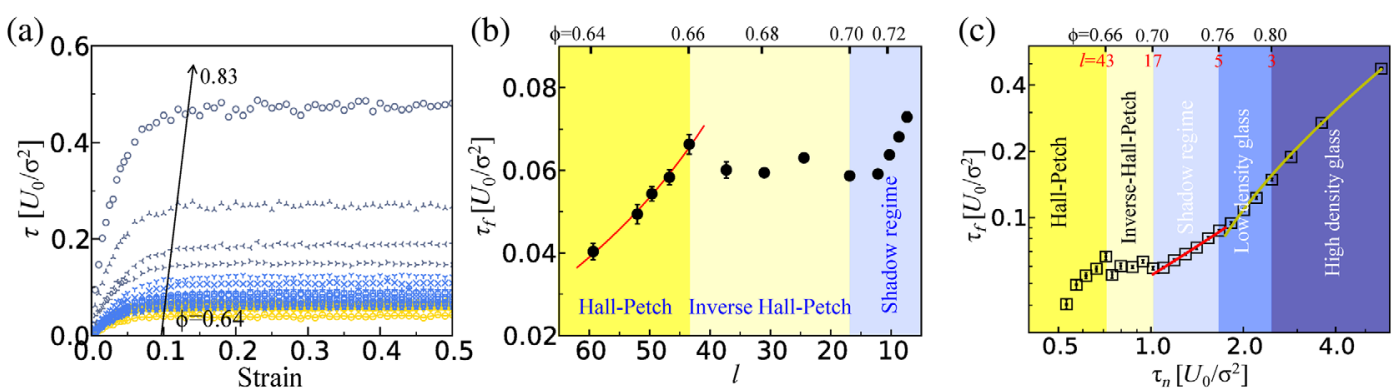

FIG. 4. (a) The stress-strain curves at different $\phi$ 's ranging from 0.64 to 0.83 with a step size of 0.005 . (b) The flow stress $\tau_{f}(l)$ shows the typical Hall-Petch and inverse Hall-Petch behaviors at $\phi \leq 0.66$ and $0.66<\phi<0.70$, respectively. $\tau_{f}(l)$ follows the standard HallPetch relationship $\tau_{f} \sim l^{-1 / 2}$ (red curve) at $\phi \leq 0.66$. (c) Log-log plot $\tau_{f}\left(\tau_{n}\right)$ shows the Mohr-Coulomb glassy behaviors with fittings $\tau_{f}=0.007+0.047 \tau_{n}$ at $0.70<\phi<0.76$ (red curve) and $\tau_{f}=-0.092+0.100 \tau_{n}$ at $\phi>0.76$ (yellow curve).

Disordered particles increase with $\phi$ and percolate in both $x$ and $y$ directions at $\phi \geq 0.70$ [Fig. 2(c)]. The percolation at $\phi=0.70$ coincides with the polycrystalglass transition, indicating that the largest cluster of disordered particles may serve as the backbone of the glass. We define neighboring disordered particles as those belonging to the same disordered cluster. The number of disordered clusters begins to decrease near $\phi=0.70$ [Fig. 2(b)], confirming the percolation at $\phi \geq 0.70$. Note that disordered particles do not percolate when grain sizes are large because many grain boundaries are low-angle grain boundaries which are chains of disconnected dislocations. The fraction of crystalline particles is $63 \%$ at $\phi=0.70$, and the mean grain diameter $l=15 \pm 2$ particles. Interestingly, this is consistent with the $3-\mathrm{nm}$ mean grain diameter of Ni-Mo [13] and Ni-W [37] alloy polycrystals, where the glassy behavior begins to emerge under plastic deformation [37]. Since $3 \mathrm{~nm}$ was the finest available grain size in Refs. [13,37], the crossover to the glass regime was not measurable. In conventional glass transition studies, small crystalline domains have been observed in certain supercooled liquids which exhibit strong correlations with a diverging length scale as approaching the glass transition point [16-18]. Here we also observed small crystalline domains in the glass regime, while the crystalline domain size continuously increases across the glass-polycrystal boundary [Fig. 1(c)].

\section{B. Mechanical properties}

\section{Equation of state}

The measured mechanical properties $\beta, K$, and $\tau_{f}$ further reveal two polycrystal regimes and three glass regimes [Figs. 3(b), 4(b), and 4(c)]. The equation of state (EOS) $P(\phi)$ (see Appendix D) in Fig. 3(a) is monotonic and without a Mayer-Wood loop [38], indicating the absence of a first-order phase transition. The peak of compressibility $\beta=\phi^{-1} d \phi / d P$ at $\phi=0.70$ shows that the system is softest at the polycrystal-glass boundary, in accordance with the strongest fluctuations in structure [Fig. 2(a)] and dynamics
[Figs. 2(d) and 2(e)] at $\phi=0.70$. We attribute the peak to the competing effects: as pressure increases, soft disks are more compressible, but fewer uncompressed disks are available.

\section{Hall-Petch and inverse Hall-Petch behaviors in the polycrystal regime}

The bulk modulus $K=\beta^{-1}$ in Fig. 3(b) clearly exhibits five regimes at different grain sizes $l$. The increase in $K(l)$ at $\phi<0.66$ and decrease at $0.66<\phi<0.70$ are similar to the Hall-Petch [39-41] and inverse Hall-Petch $[11,13,37,42]$ behaviors, respectively. In the Hall-Petch (or inverse Hall-Petch) behavior, the mechanical strength or the ability to resist plastic deformation increases (or decreases) as the polycrystalline grains become finer. Usually, polycrystals exhibit the Hall-Petch behavior at a grain size $l \gtrsim$ $15 \mathrm{~nm}$ and the inverse Hall-Petch behavior at $l \lesssim 15 \mathrm{~nm}$ $[11,13]$; it is not clear whether and how the inverse HallPetch behavior terminates at a very small grain size. Whether glasses exhibit Hall-Petch or inverse Hall-Petch behavior has not been studied before because the crystalline grain size is not defined in most glasses. Since the HallPetch relation is typically expressed in terms of the flow stress $\tau_{f}[11,42]$ (see Appendix E), we measure $\tau_{f}(l)$ under plastic deformation [Fig. 4(a)], which can be well fitted by the 3D Hall-Petch relation $\tau_{f} \sim l^{-1 / 2}$ at $\phi \leq 0.66$ [red curve in Fig. 4(b)]. At $\phi>0.66, \tau_{f}(l)$ deviates from the HallPetch relation and the inverse Hall-Petch behavior emerges. Interestingly, the grain diameter $l$ at the onset of the inverse Hall-Petch regime (i.e., $\phi=0.66$ in our $2 \mathrm{D}$ system) is approximately $43 \sigma$, which agrees very well with the measured grain diameter of $10-15 \mathrm{~nm}$ (i.e., $l \simeq 38-57 \sigma$ ) at the onset of the inverse Hall-Petch behavior in 3D Cu [11] and $\mathrm{Ni}[13,37]$ polycrystals. Note that $\mathrm{Cu}$ and $\mathrm{Ni}$ are most popularly used in the studies of the inverse Hall-Petch behavior since they can form fine-grained polycrystals. The Hall-Petch and inverse Hall-Petch behaviors have usually been studied in 3D, while we find that they also hold in $2 \mathrm{D}$. 


\section{Mohr-Coulomb relation in glass regimes}

In granular and metallic glasses, $\tau_{f}$ and the normal stress $\tau_{n}$ satisfy the Mohr-Coulomb relation [6], $\tau_{f}=\tau_{0}+\alpha \tau_{n}$, where $\tau_{0}$ is a constant and $\alpha$ is the effective friction coefficient. The linearly increasing regimes of $\tau_{f}\left(\tau_{n}\right)$ at $0.70<\phi<0.76$ and $\phi>0.76$ in Fig. 4(c) represents different glassy responses to plastic deformation [6]. The smaller $\alpha=0.047$ at $0.70<\phi<0.76$ than the fitted $\alpha=$ 0.100 at $\phi \geq 0.76$ is consistent with the small bulk modulus [Fig. 3(b)] and the strong dynamics [Figs. 2(d) and 2(e)] in this regime. Such a shadow glass regime characterized by strong dynamics near the glass transition point has been observed in metallic glasses [43].

\section{Thermodynamic properties}

\section{Density of state}

In solids, structural and mechanical properties are connected through the vibrational DOS $[24,44,45]$. We measure the vibrational modes from the covariance matrix of particle displacements and derive the DOS [24,44,45] (see Appendix F). The high elastic modulus of high-density glasses [Fig. 3(b)] leads to more high-frequency modes as shown in Figs. 5(a) and 5(b). The high-frequency part in the semilog plot [Fig. 5(b)] is concave in the polycrystal regime and convex in the glass regime, indicating that vibrational modes have broader distributions in glasses. This is in accordance with the broad distributions of relaxation in glassy systems associated with dynamic heterogeneities
[1]. Interestingly, the frequency $\omega_{\max }$ of the DOS peak and its derivative in Fig. 5(c) exhibit almost the same shape as $P(\phi)$ and its derivative in Fig. 3(a). The DOS peaks show a power law in Fig. 5(a), which has rarely been reported in other systems. These peaks become the densest at $\phi=0.70$, as quantified in Fig. 5(d). The step sizes $\Delta \mathrm{DOS}_{\max }$ and $\Delta \omega_{\max }$ reach their respective minima at $\phi=$ 0.70 [Fig. 5(d)]. Higher pressures shift modes to higher frequencies as Fig. 5(a) shows, while the rate of such a shift reaches a minimum at $\phi=0.70$ [Fig. 5(d)], corresponding to the softest solid shown in Fig. 3(b). $\Delta \omega_{\max }(\phi)$ [Fig. 5(d)] exhibits a similar curve shape to that of $K(\phi)$ [Fig. 3(b), especially when it is plotted in linear scale] and can clearly distinguish the five regimes, indicating that the five regimes have different behaviors of vibrational modes.

\section{Specific heat}

We further measure the thermodynamic quantity $c_{V}=\left(\left\langle E^{2}\right\rangle-\langle E\rangle^{2}\right) /\left(N k_{B} T^{2}\right)$, where $E$ is potential energy. We find that $c_{V}$, i.e., the energy fluctuation, peaks at $\phi=$ 0.70 [Fig. 6(a)], in accordance with the strongest fluctuations in structure [Fig. 2(a)] and dynamics [Figs. 2(d) and 2(e)]. $c_{V}$ exhibits distinct behaviors in polycrystals and glasses. In the polycrystal regime, both $c_{V}(\phi)$ in Fig. 6(a) and the density of disordered particles in Fig. 6(b) increase quadratically, suggesting that disordered particles contribute most to $c_{V}$. This relation between $c_{V}$ and disorderness agrees with our observation that the dynamics of disordered particles are stronger than those of crystalline particles
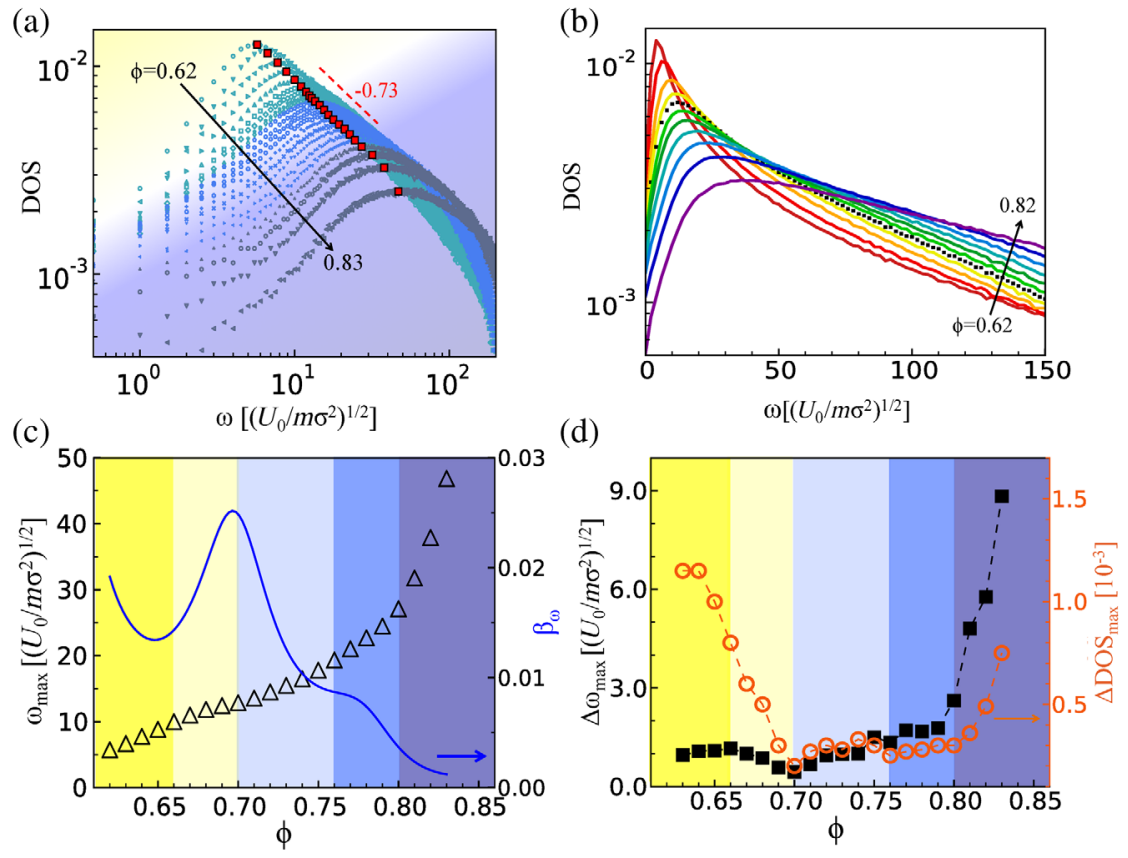

FIG. 5. (a) The DOS peaks (red squares) follow the relation $\operatorname{DOS}_{\max } \sim \omega_{\max }^{-0.73}$. (b) In the semilog plot of DOS, the tail is convex in the polycrystal regime, convex in the glass regime, and straight at the crystal-glass boundary $\phi=0.70$ (black symbols). (c) The frequency of the DOS peak, $\omega_{\max }$ (triangles), has a similar shape to $P(\phi)$ in Fig. 3(a). $\beta_{\omega} \equiv \phi^{-1} d \phi / d \omega$ (blue curve) has a similar shape to that of the compressibility in Fig. 3(a). (d) The step size $\Delta \mathrm{DOS}_{\max }$ (circles) and $\Delta \omega_{\max }$ (squares) between neighboring peaks. 

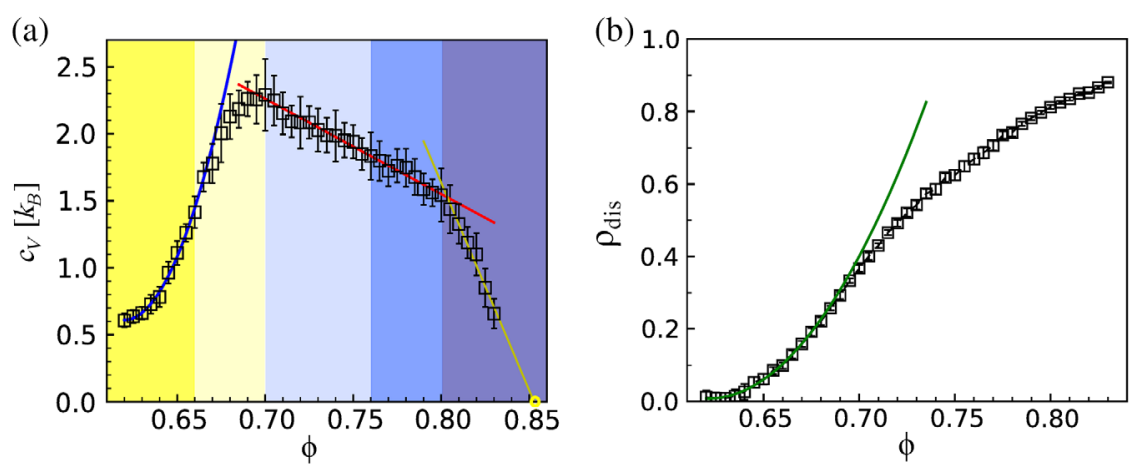

FIG. 6. (a) Residual specific heat $c_{V}$ with a quadratic fit [blue curve, $c_{V}=515.6(\phi-0.6197)^{2}+0.608$ ] in the polycrystal regime and two linear fits (red and yellow lines) in the glass regimes. The linear extrapolation yields $c_{V}(\phi=0.853)=0$ marked by the yellow circle. (b) The fraction of disordered particles with a quadratic fit $\rho_{\text {dis }}=62.95(\phi-0.621)^{2}+0.0081$ (green curve) in the polycrystal regime $\phi<0.70$. The two quadratic fits in (a) and (b) with the same minimum $\phi \simeq 0.62$ show that $c_{V} \propto \rho_{\text {dis }}$.

[Figs. 1(e) and 1(f)]. Similar behaviors have been observed in metals, whose density of vacancies determines $c_{V}$ [46]. $c_{V}(\phi)$ deviates from the quadratic fitting near the polycrystal-glass boundary $\phi=0.70$, where the disordered particles begin to percolate [Fig. 2(c)]. This percolation may suppress the fluctuation of disordered particles and thus reduce $c_{V}$ at $\phi>0.70$.

In the glass regimes, $c_{V}$ decreases with $\phi$ [Fig. 6(a)]. $c_{V}$ of glasses and polycrystals have mainly been studied as a function of $T$ and rarely as a function of density. Reference [47] reported $c_{V}$ of silica glass at two densities and a lower $c_{V}$ at the higher density, in accordance with our observation. Our measured $c_{V}(\phi)$ exhibits two separate linear regimes for the high-density and low-density glasses. We attribute the linear behavior to the linear decrease in free volume as $\phi$ increases. The linear extrapolation to $c_{V}=0$ yields $\phi=0.853$ [Fig. 6(a)], where particles stop vibrating due to their close packing, in agreement with the 2D random close packing $\phi_{\text {rcp }} \simeq 0.847$ [48].

\section{Glass-glass transition}

When particles interact on two length scales (e.g., the shoulder potential), they can form low-density and highdensity glasses [35,49], corresponding to the states in which particles are mainly caged by long-scale and short-scale interactions [35,50]. Such local cages induce the dynamics arrest of glasses [35,51]. One feature of the glass-glass transition is that the second peak of $g(r)$ becomes higher than the first peak, which reflects a change in the local cage structure [35]. This feature can be seen in our system at $\phi=0.80$, labeled by a dashed line in Fig. 1(d). In addition, we discover new features at this glass-glass transition, including changes in the slopes of $\beta$ [Fig. 3(a)], $K$ [Fig. 3(b)], $c_{V}$ [Fig. 6(a)], and the properties related to the DOS [Figs. 5(c) and 5(d)]. These features show that the high-density and low-density glasses exhibit different mechanical and thermodynamic behaviors.

\section{HOW TO PRODUCE ULTRAFINE-GRAINED POLYCRYSTALS}

Adding atoms of different sizes, i.e., solutes, is the most popular method to reduce the grain size and improve the stability of ultrafine-grained polycrystals $[5,13]$. The mean grain size can be reduced to $\sim 3 \mathrm{~nm}$ (i.e., the grain diameter $l \simeq 12$ particles) by adding $\sim 20 \%$ solutes $[13,37]$. The presence of more solutes often leads to vitrification and amorphous solids without a measurable crystalline grain size [29]. Stable ultrafine grains $(l<12$ particles) were not available in previous studies, and thus it was difficult to explore the polycrystal-glass transformation. In our system, the "solute particles" (i.e., the compressed soft particles) can be continuously "added" through compression (Fig. S1 of Supplemental Material [52]). Direct vitrification can be avoided because the solutes are not added to the liquid solution all at once but are "incrementally added" to the polycrystal. The compressed solid matrix helps to suppress grain coarsening. These two effects can continuously reduce the grains to an extremely small size [Fig. 1(c)]. By contrast, the conventional methods of fabricating finegrained polycrystals using the ball-milling method [5] or dc electrodeposition in solution [13] are less effective because the former uses samples with a limited amount of solute and the latter proceeds in a liquid environment which facilitate crystallization and grain coarsening.

We similarly simulate binary systems in a broader parameter space with different shoulder-potential widths $\lambda$ and different fractions of soft particles $\eta$. Glasses cannot form when $\lambda$ and $\eta$ are too small (see the orange region in Fig. 7). For example, a system with $\lambda=1.28$ and $\eta=0.25$ produces the minimum $l=11$, which is a polycrystal instead of a glass (Fig. 8). To achieve ultrafine-grained polycrystals and access the polycrystal-glass boundary, both $\lambda$ and $\eta$ must be sufficiently large to produce sufficient amounts of solutes and create a size mismatch. In the fabrication of metallic glasses, sufficient atomic volume 


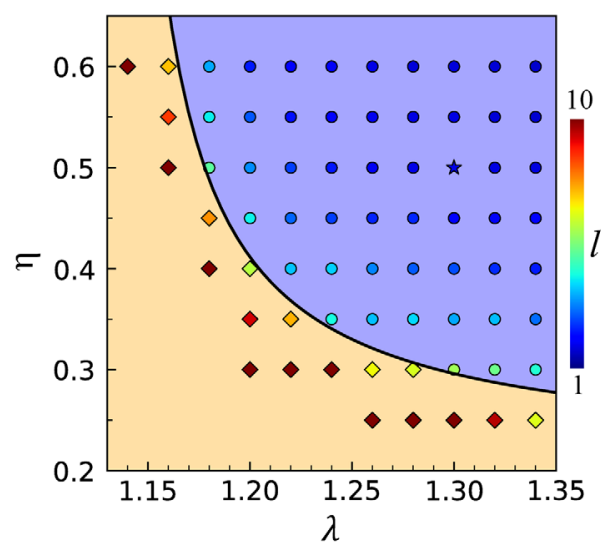

FIG. 7. The finest mean grain diameter $l$ for systems with different $\eta$ 's and $\lambda$ 's. $(\eta-0.193)(\delta-0.214)=0.02$ (solid curve) separates the blue region exhibiting a polycrystal-glass transition (circles) and the orange region exhibiting no polycrystal-glass transition (diamonds). The star represents the system used in Figs. 1-6.

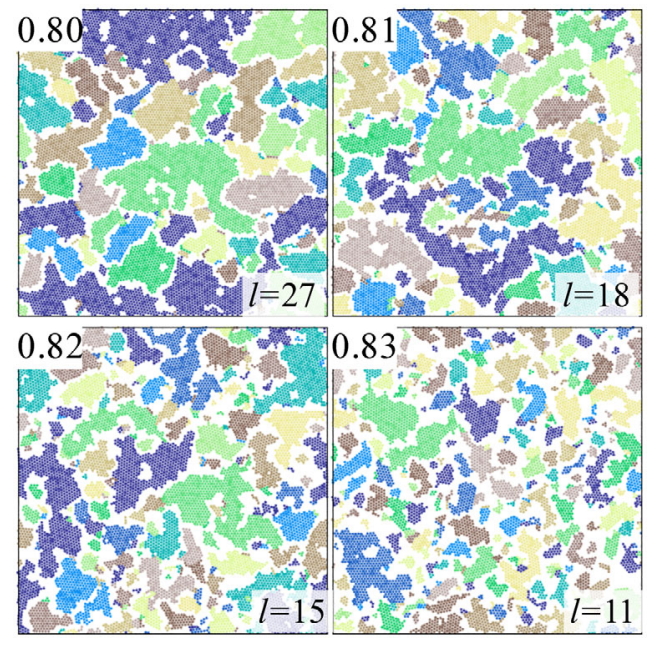

FIG. 8. Polycrystalline structures at $\phi=0.80,0.81,0.82$, and 0.83 for the system with shoulder width $\lambda=1.28$ and soft particle fraction $\eta=0.25$. Its $\left(\eta-\eta_{0}\right)\left(\lambda-\lambda_{0}\right)<0.02$; hence the system remains in a polycrystal instead of a glass at the highest $\phi$. Note that it is difficult to compress the system beyond $\phi=0.83$, which is near the random close packing $\phi_{\text {rcp }}=0.847$ of binary disks.

mismatch $\delta=\Delta V / V_{A}$ is required, where $\Delta V=V_{A}-V_{B}$ [29]. $V_{A}$ is the volume per atom in the matrix, and $V_{B}$ is the volume per solute atom. Here we find that the region exhibiting a polycrystal-glass transformation is located at $\left(\eta-\eta_{0}\right)\left(\delta-\delta_{0}\right)>0.02$ (blue region in Fig. 7), where $\delta=\left[\left(\lambda^{2}-1\right) / \lambda^{2}\right], \quad \eta_{0}=0.193 \pm 0.002, \quad$ and $\delta_{0}=0.214 \pm$ 0.002 . This is similar to the empirical relation showing that the minimum solute concentration needed to form a glass is roughly inversely proportional to $\delta$ [29]. Moreover, we find that there exist minimum thresholds $\eta_{0}$ and $\delta_{0}$ for glass formation.
Figure 7 provides guidance on fabricating ultrafinegrained polycrystals which could break the current limit of $l \simeq 3 \mathrm{~nm}$ in atomic systems: (1) using a "soft-hard" binary system, (2) increasing the pressure to incrementally add the "solutes" to the polycrystal, and (3) having plenty of soft particles for generating sufficient solutes and soft enough particles to create a large size mismatch at high pressures. Metallic glasses have been achieved by adding sufficient size-mismatched solutes [29], but ultrafine-grained metallic polycrystals have not been obtained because conditions (1)-(3) are seldom satisfied simultaneously.

We observe that the polycrystal-glass boundary depends on system parameters. For example, it shifts to a lower $\phi$ as $\eta$ increases at $\lambda=1.30$. Systems in the blue region of Fig. 7 can be compressed into glasses, and their mean grain size at the polycrystal-glass boundary decreases from $l=21$ to 10 as $\eta$ increases from 0.40 to 0.60 .

\section{POLYCRYSTAL-GLASS TRANSITION IN 3D}

We compress 3D crystals to completely disordered glasses [Fig. 9(a)] from $\phi=0.44$ to 0.62 at $T=$ $0.2 U_{0} / k_{B}$. The system is composed of 5000 hard spheres and 5000 soft spheres with shoulder potentials shown in Fig. 1(a). The fraction of disordered particles increases rapidly at $\phi=0.48$ [Fig. 9(b)], suggesting a sharp polycrystal-glass transition. This transition point is further confirmed by the peaks of $\beta, \chi_{6}, \operatorname{RSD}(L)$, and $c_{V}$ in Figs. 9(c) -9 (f). These peaks in 3D systems have also been observed in the 2D systems in Figs. 2(a), 2(d), 3(a), and 6(a); hence, they are robust features at the polycrystalglass transitions in our 2D and 3D systems. Similar to the EOS of 2D systems, the EOS of 3D systems is monotonic without a Mayer-Wood loop [Fig. 9(c)], indicating that it is not a first-order transition. At the polycrystal-glass boundary $\phi=0.48$, the average 3D grains contain about 4000 particles, which is comparable to the system size. Hence, the 3D system size is not enough to show how quantities change with the grain size such as the Hall-Petch behavior. Nevertheless, the system size is large enough to clearly resolve the crystalglass boundary and its associated features. In fact, we found that a system size of 800 particles is enough to clearly show the peak of the compressibility [Fig. 13(a)].

The crystalline order of particles in $3 \mathrm{D}$ is characterized by the modified bond-orientational order parameter $q_{6}$ [53] (see Appendix B). Particles with low disorderness $\left(q_{6}<0.35\right)$ and strong dynamics $(L>0.20)$ are highlighted in Figs. 10(a)-10(c) and Figs. 10(d)-10(f), respectively. Particles with strong dynamics [Figs. 10(d) and 10(e)] are mainly disordered ones [Figs. 10(a) and 10(b)] in polycrystals at $\phi<0.48$, but disperse randomly in glasses [Figs. 10(c) and 10(f)]. This is consistent with the observations in 2D polycrystal-glass transition in Fig. 1. During compression, disordered particles abruptly increase [Fig. 9(b)]. Unlike 2D systems where the high-density glass possesses tiny crystalline clusters [Fig. 1(i)], the binary 3D crystals can be 


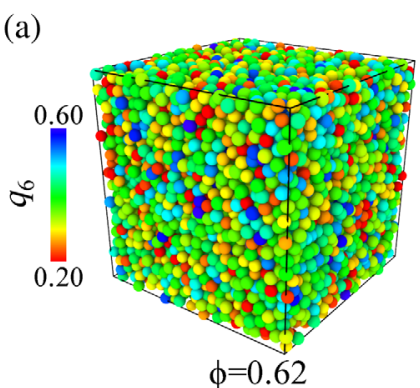

(d)

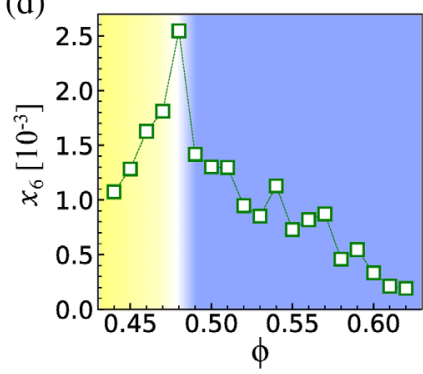

(b)

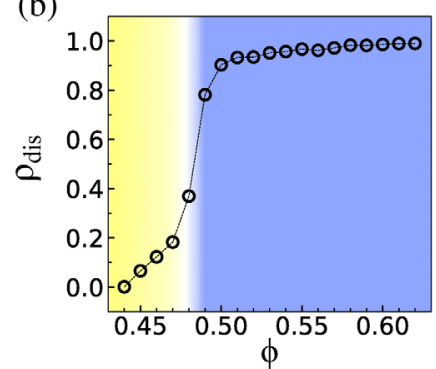

(c)

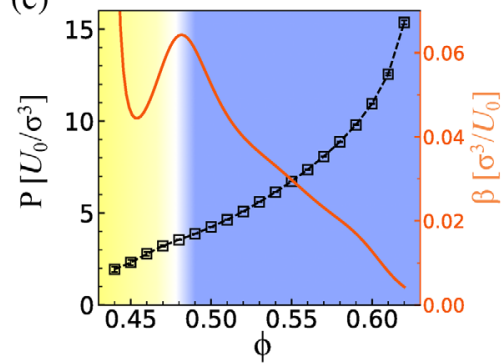

(e)

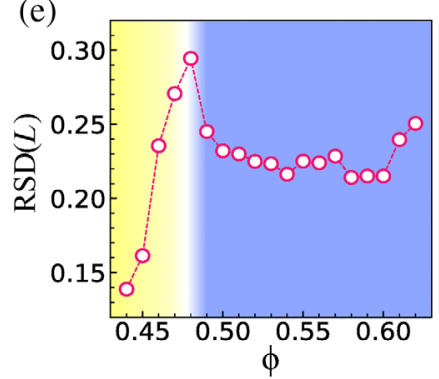

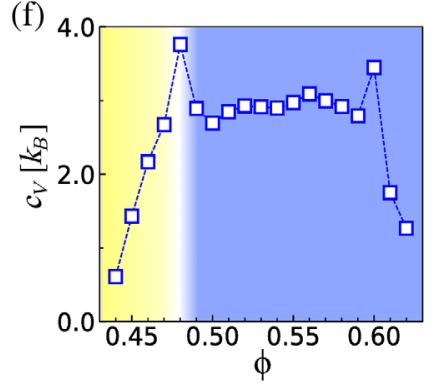

FIG. 9. The compression-induced crystal-glass transition in 3D. (a) The completely disordered glass state at $\phi=0.62$ colored with weighted bond-orientational order $q_{6}$. (b) The fraction of disordered particles. (c) The EOS $P(\phi)$ and compressibility $\beta$. (d) Susceptibility $\chi_{6}$ of the 3D orientational order. (e) Relative standard deviation of $(L)$. (f) The residual specific heat $c_{V}$.

(a)

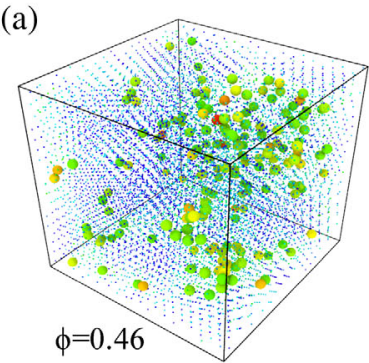

(d)

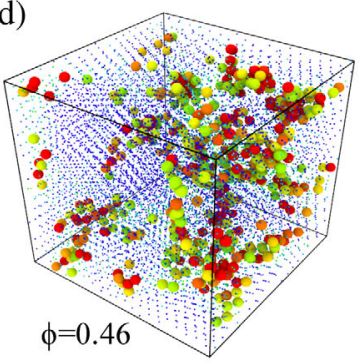

(b)

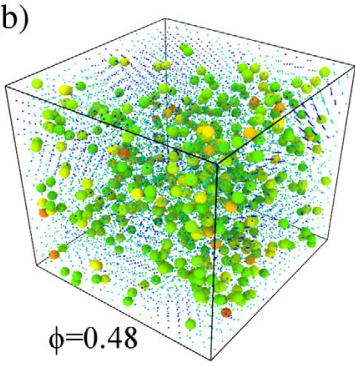

(e)

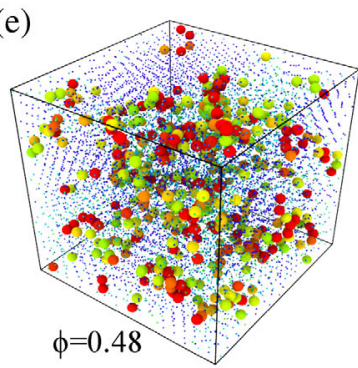

(c)

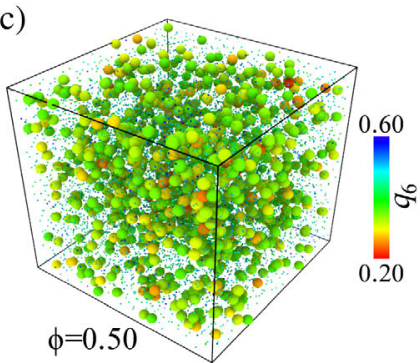

(f)

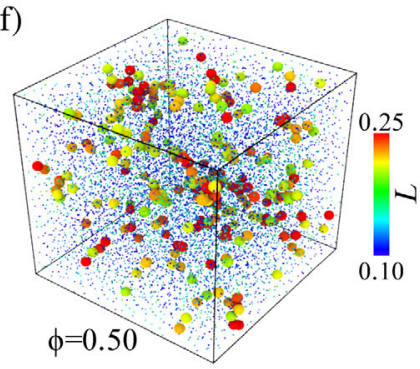

FIG. 10. (a)-(c) Weighted bond-orientational order parameter $q_{6}$ and (d)-(f) Lindemann parameter $L$ at $\phi=0.46,0.48$, and 0.50 , respectively. Particles with $q_{6}>0.35$ and $L<0.20$ are shown as the small blue dots for the better visualization of disordered (i.e., small $q_{6}$ ) and strong moving (i.e., large $L$ ) particles.

compressed into a completely disordered glass [Fig. 9(a)], which agrees with the observations that the structural correlation length in 3D glassy systems is much smaller than that in 2D [18].

\section{DISCUSSION AND CONCLUSION}

In this work, we study the following four questions which have not been answered in literature. (1) How can we create stable ultrafine-grained polycrystals with a mean grain diameter $l<12$ particles? We achieved such stable ultrafine-grained polycrystals in $2 \mathrm{D}$ and gave their empirical formation criterion $(\eta-0.193)(\delta-0.214)>0.02$ in Sec. IV. The particle-size-mismatch mechanism for the formation of ultrafine-grained polycrystals is discussed in Sec. IV. (2) Given an ultrafine-grained polycrystal, should we call it a polycrystal or a glass, or is it simply a matter of terminology without basic importance? (3) Is the 
polycrystal-glass transition a crossover or a sharp transition? (4) What are the features at the transition? These questions have rarely been asked or studied before. We observed a sharp polycrystal-glass transition; thus, whether an ultrafine-grained polycrystal should be called a polycrystal or a glass is not just a matter of terminology. The observed rich features at the transition cast new light on the open question of how to rigorously define polycrystals, glasses, or amorphous solids.

We applied those analysis methods mainly used for glass studies [Figs. 4(c), 5(a), and 14] and those mainly used for polycrystal studies [Figs. 1(c), 3, 4(a), and 4(b)] to both glass and polycrystal regimes. In addition, we used new analysis methods in Figs. 2(b), 2(d)-2(f), and 5(b)-5(d). Surprisingly, different types of features from these analyses coincide at the same $\phi$, indicating that the polycrystal-glass transition occurs at a sharp boundary rather than a gradual crossover. Many other analyses in the liquid-glass transition can be similarly applied to such a crystal-glass transition in the future.

Besides the major results about the polycrystal-glass transition, the analyses provide new results about the previously studied phenomena of Hall-Petch behavior and the glass-glass transition. New features are observed at the Hall-Petch to inverse Hall-Petch transition [Figs. 2, 3, 5(c), and 5(d)], the shadow glass transition [Figs. 2(b), 2(d), and 2(e)], and the glass-glass transition [Figs. 3(b), 5(d), and 6(a)], which cast light on their mechanisms. In fact, whether the inverse Hall-Petch behavior exists in 2D materials has not been well studied. We found that it exists in $2 \mathrm{D}$ as it does in 3D [11,13]. In addition, we observed for the first time that the well-known inverse Hall-Petch behavior terminates at a fine enough grain size.

In 2D solids, we observed similar features to those of 3D solids, such as the Hall-Petch [39-41] and inverse HallPetch $[11,13,37,42]$ behaviors in polycrystals, the MohrCoulomb relation in glasses [6], the structural features at the glass-glass boundary [35], and $c_{V}$ linearly increasing with the defect density in the crystal regime [46]. In fact, we did not observe any dissimilar features to those of 3D polycrystals or glasses shown in the literature. On the other hand, lower-dimensional solids are much "softer" because particles are less constrained by neighbors. Consequently, the dimensionality may strongly affect the nature of phase transitions [54] and glass properties [7,55]. For example, 2D crystals often melt via a two-step transition rather than a one-step melting transition as in 3D crystals [56,57]. Glassy systems in 2D and 3D share many similarities, such as dynamic heterogeneities [58] and the nature of glass transition [59], but they also differ in terms of spatial fluctuations and relaxations $[7,9,10]$. We further simulated the same binary system in 3D and found that the susceptibility of orientational order, fluctuation of the Lindemann parameter, compressibility, and residual specific heat all peak at the same $\phi$, indicating that the sharp crystal-glass transitions exist in both $2 \mathrm{D}$ and $3 \mathrm{D}$ and have similar features. It may be possible to use the features at the polycrystal-glass boundary observed in these binary systems to distinguish polycrystals and glasses in other systems.

The maxima or minima at the polycrystal-glass boundary in Figs. 2, 3, 4(c), 5(c), 5(d), and 6(a) could hold in real materials. The minimum flow stress [Fig. 4(c)] and the highest compressibility [Figs. 3(a) and 9(c)] and residual specific heat [Figs. 6(a) and 9(f)] could be easily tested experimentally. In fact, similar trends on both sides of the polycrystal-glass boundary have been experimentally observed for 3D solids in separate studies. For example, the inverse Hall-Petch behavior in Fig. 4(b) and the MohrCoulomb behavior in Fig. 4(c) have been observed in 3D solids, but they have not been put alongside each other in the same plot, as is done in Fig. 4(c), since the crystalline grain size is poorly defined in most glasses. For another example, it is known that the heat capacity increases with the defect density in numerous 3D crystalline metals, decreases with density in 3D glasses, and vanishes at the random close packing, but the possible peak at the polycrystal-glass boundary [Fig. 6(a)] has not been explored because the defect density is not well defined in glasses.

The pressure-induced crystal-glass transition likely occurs widely in binary systems as long as one type of particles is softer than the other and the pressure is high enough. For example, our further simulations showed that binary systems with hard and soft Weeks-ChandlerAndersen potentials or inverse power-law potentials exhibit similar polycrystal-glass transitions. Such soft-hard binary systems under compression provide an interesting platform to study various questions in glasses and polycrystals. The modern high-pressure technique [60] can deform the diameter of a soft atom such as cerium by 20\% [61], and thus we expect to be able to compress certain alloys into ultrafine-grained polycrystals and metallic glasses. Such a polycrystal-glass transition would be more easily achieved in colloidal, micelle, and granular systems whose particles can be easily deformed.

\section{ACKNOWLEDGMENTS}

We thank Marcus Bannerman, Bruce D. Gaulin, Patrick Charbonneau, and Peng Tan for helpful discussions. This work was supported by RGC Grants No. GRF601613 and No. A-HKUST616/14 and Shenzhen Grant No. JCYJ20170307174000693.

\section{APPENDIX A: SIMULATIONS}

We performed event-driven molecular dynamics simulations [62] in the 2D NAT ensemble (i.e., constant particle number $N$, area $A$, and temperature $T$ ) with periodic boundary conditions. For $N / 2$ hard disks and $N / 2$ soft disks in a square box with side length $L_{\mathrm{box}}$, the area fraction 


$$
\phi \equiv \frac{N}{2} \frac{\pi \sigma^{2}}{L_{\mathrm{box}}^{2}} \frac{\left(1.3^{2}+1^{2}\right)}{4} .
$$

The soft disks have an inner hard core with unit diameter $\sigma$, which is used in the calculation of $\phi$. The 2D crystal has many defects at $T>0.2 U_{0} / k_{B}$, and is almost frozen with little dynamics at $T<0.05 U_{0} / k_{B}$. We therefore chose an intermediate temperature of $T=0.133 U_{0} / k_{B}$. Disks were randomly distributed on the lattice at $\phi=0.62$, and then relaxed at $T=1.333 U_{0} / k_{B}$ for a time period of $10^{5} t_{0}$, at $T=0.667 U_{0} / k_{B}$ for $10^{5} t_{0}$, and finally at $T=0.133 U_{0} / k_{B}$ for $10^{5} t_{0} \cdot t_{0}=\sqrt{m \sigma^{2} / U_{0}}$ is the amount of time a disk takes to move a distance $\sigma$, where $m$ is the mass of both types of disks. Different initial velocities following Gaussian distributions were assigned every $10^{4} t_{0}$ to facilitate the equilibration [19]. At $\phi=0.62$ and $T=$ $0.133 U_{0} / k_{B}$, the crystal was nearly defect-free (Fig. 11). This crystal was consequently compressed into higher area fractions quasistatically by $\Delta \phi=0.005$ per step using the Lubachevsky-Stillinger algorithm [63]. At each $\phi$, the sample is relaxed for $10^{5} t_{0}$ steps followed by another $10^{4} t_{0}$ steps to produce data. The quantities do not vary over time, indicating sufficient equilibration (see Supplemental Material, Fig. S2 [52]). We focused on the range from a single crystal at $\phi=0.62$ to a glass at $\phi=0.83$, which is near the close packing $\phi_{\mathrm{cp}} \simeq 0.847$ [48]. The results in Figs. 1(c), 2(b), 2(f), 3, 4, and 6 were averaged over 20 independent simulations for reliable statistics. Other results in Figs. 1, 2, 5, and 6 are obtained from one representative compression process. No particle swapping occurs at each $\phi$ (see Videos S1-S3 in Supplemental Material [52]), confirming that the disordered system is solidlike, i.e., a glass, rather than a liquid [52].

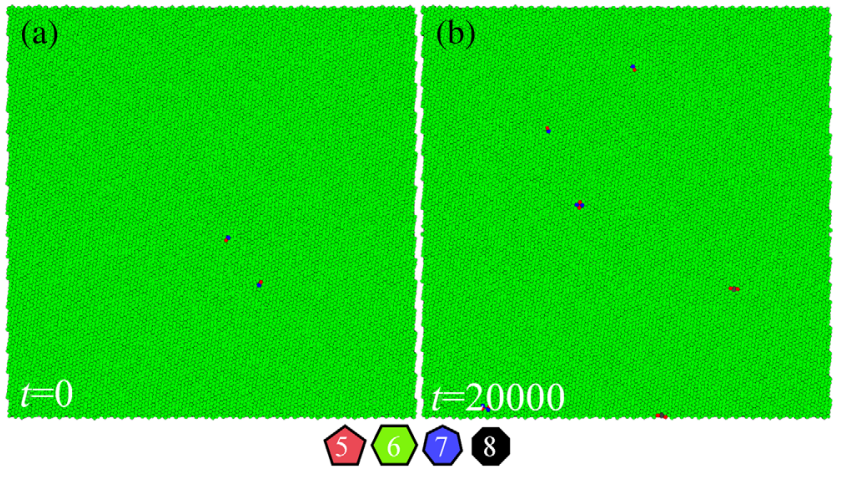

FIG. 11. The Voronoi diagrams of the single crystal at $\phi=0.62$ and $T=0.133 U_{0} / k_{B}$. Five-neighbor particles (red) and sevenneighbor particles (blue) form pairs, i.e., dislocations [64], which can easily diffuse under this temperature, as shown in (a) $t=0$ and (b) $t=20000 t_{0}$.

\section{APPENDIX B: IDENTIFICATION OF CRYSTALLINE GRAINS}

The orientational order parameter $\psi_{6 j}=\sum_{k=1}^{N_{j}} l_{j k} e^{i 6 \theta_{j k}} /$ $l_{\text {tot }}$ [38], where $\theta_{j k}$ is the orientational angle of the bond between particle $j$ and its neighbor $k$. The Voronoi polygon [65] has $N_{j}$ edges with perimeter $l_{\text {tot }}$, and the length of the edge between $j$ and $k$ is $l_{j k}$. A higher $\left|\psi_{6 j}\right|$ represents a higher crystalline order. We defined a particle with three or more crystalline bonds (i.e., the bond between particles $j$ and $k$ satisfies $\left.\left|\psi_{6 j} \cdot \psi_{6 k}^{*}\right|>0.6\right)$ as crystalline [66]. Two neighboring crystalline particles belong to the same grain if the difference between their orientational angle $\mid \operatorname{Arg}\left(\psi_{6 j}\right)-$ $\operatorname{Arg}\left(\psi_{6 k}\right) \mid \leq 4.2^{\circ}$. Noncrystalline particles and single isolated crystalline particles are defined as disordered.

To characterize the crystalline order of each particle in 3D systems, we used a modified bond-orientational order parameter in which each bond is weighed by its corresponding Voronoi facet area [53]:

$$
q_{l}(i)=\sqrt{\frac{4 \pi}{2 l+1} \sum_{m=-l}^{l}\left|\sum_{j=1}^{n} \frac{A_{j}}{A} Y_{l m}\left(\theta_{j}, \phi_{j}\right)\right|^{2}},
$$

where $\theta_{j}$ and $\phi_{j}$ are the spherical angles of the vector from particle $i$ to its $j$ th Voronoi neighbor. $A_{j}$ is the area of the Voronoi facet to the $j$ th neighbor. $A$ is the total surface area of the Voronoi cell. $Y_{l m}$ is a spherical harmonic function of degree $l$ and order $m$. Here we use $q_{l=6}$, which can distinguish disordered particles $\left(q_{6}<0.35\right)$ from crystalline ones $[17,53,67]$. The averaged $\left\langle q_{6}\right\rangle$ is used to calculate the susceptibility of orientational order in 3D system. Based on the criterion of ten Wolde and Frenkel [68], a particle with six or more crystalline bonds (i.e., the bond between neighboring particles $j$ and $k$ satisfies $\left.\left|q_{6 j} \cdot q_{6 k}^{*}\right|>0.5\right)$ is defined as crystalline. Neighboring crystalline particles belong to the same cluster, and the rest is disordered.

The orientational susceptibility [Fig. 2(a)] [69],

$$
\chi_{6}=N\left(\left\langle\bar{\psi}_{6}^{2}\right\rangle-\left\langle\bar{\psi}_{6}\right\rangle^{2}\right),
$$

where the global orientational order parameter $\bar{\psi}_{6}$ is the $\left|\psi_{6 j}\right|$ averaged over all particles in a frame. \langle\rangle represent time average. The averaged $\left|q_{6}\right|$ is used in the same formula [Eq. (B2)] to calculate the 3D susceptibility of the orientational order $\chi_{6}$.

Since there are numerous tiny grains, it is more reasonable to define the mean grain diameter as the weighted grain size [70],

$$
l \equiv \sqrt{N_{g}}=\left(\sum n_{s} s^{2} / N_{c}\right)^{1 / 2},
$$

where $N_{g}$ is the weighted mean number of crystalline particles per grain, $n_{s}$ is the number of grains with $s$ 
crystalline particles, $N_{c}$ is the total number of crystalline particles, and $n_{s} s / N_{c}$ is the probability that a particle belongs to the $s$-sized grains. $l(\phi)$ in Fig. 1(c) decreases nearly exponentially with the steepest slope at $\phi=0.70$.

\section{APPENDIX C: DYNAMICS}

The Lindemann parameter $L$ is the ratio of the vibrational amplitude to the lattice constant. Since the lattice constant is ambiguous in glasses, we use the diameter $\sigma$ of the inner core of the soft disk in the definition:

$$
L_{j} \equiv \sqrt{\left\langle\mathbf{r}_{j}^{2}\right\rangle-\left\langle\mathbf{r}_{j}\right\rangle^{2}} / \sigma,
$$

where $\mathbf{r}_{j}$ is the position of particle $j$ and \langle\rangle denotes the average over $400 t_{0}$, which corresponds to the plateau in the mean-square displacement. The relative standard deviation of $L$ [Fig. 2(d)],

$$
\operatorname{RSD}(L)=\sqrt{\left\langle L^{2}\right\rangle-\langle L\rangle^{2}} /\langle L\rangle .
$$

To characterize how rugged the landscape of $L$ is, we propose to measure the variance of $\Delta L$,

$$
\operatorname{Var}(\Delta L)=\left\langle(\Delta L)^{2}\right\rangle-\langle|\Delta L|\rangle^{2},
$$

where $\Delta L=L_{k}-L_{j}$ is the difference in $L$ between neighboring particles.

The correlation between the dynamics and structural disorder,

$$
\begin{aligned}
C_{\mathrm{dis}-L} & =\left\langle\left(1-\left|\psi_{6 j}\right|\right) L_{j}\right\rangle-\left\langle 1-\left|\psi_{6 j}\right|\right\rangle\left\langle L_{j}\right\rangle \\
& =\left\langle\left|\psi_{6 j}\right|\right\rangle\left\langle L_{j}\right\rangle-\left\langle\left|\psi_{6 j}\right| L_{j}\right\rangle,
\end{aligned}
$$

where $1-\left|\psi_{6 j}\right|$ represents the disorder of a particle and \langle\rangle is ensemble average.

\section{APPENDIX D: EQUATION OF STATE}

The stress tensor $\mathbf{P}=\mathbf{P}_{v}+\mathbf{P}_{i}$, where the velocity term $\mathbf{P}_{v}=(1 / V) \sum_{j=1}^{N} m \mathbf{v}_{j} \mathbf{v}_{j}$ and the interaction term $\mathbf{P}_{i}=$ $(1 / V) t_{\text {sim }} \sum_{j=1}^{N_{\text {event }}} \Delta \mathbf{p}_{j} \mathbf{R}_{i j}$ [62]. The simulation time $t_{\text {sim }}=10000 \tau_{0}$. The summation is performed over $N_{\text {event }}$ collision events between all particle pairs. $\Delta \mathbf{p}_{j}$ is the momentum impulse on particle $j . \mathbf{R}_{i j}$ is the relative position of two colliding particles. The hydrostatic pressure is calculated from the pressure tensor $P=\left(P_{x x}+P_{y y}\right) / 2$ for $2 \mathrm{D}$ and $P=\left(P_{x x}+P_{y y}+P_{z z}\right) / 3$ for $3 \mathrm{D}$. We fitted the EOS $P(\phi)$ with a seventh-order polynomial and obtained its derivative for calculating the compressibility [71] in Fig. 3(a). The 2D compressibility $\beta \equiv-\{(1 / A)[(d A) /(d P)]\}=$ $(1 / \phi)[(d \phi) /(d P)]$, where the area $A \propto \phi^{-1}$. The EOS and $\beta$ at different system sizes in Figs. 12 and 13 show that the finite-size effect is small when $N>800$.
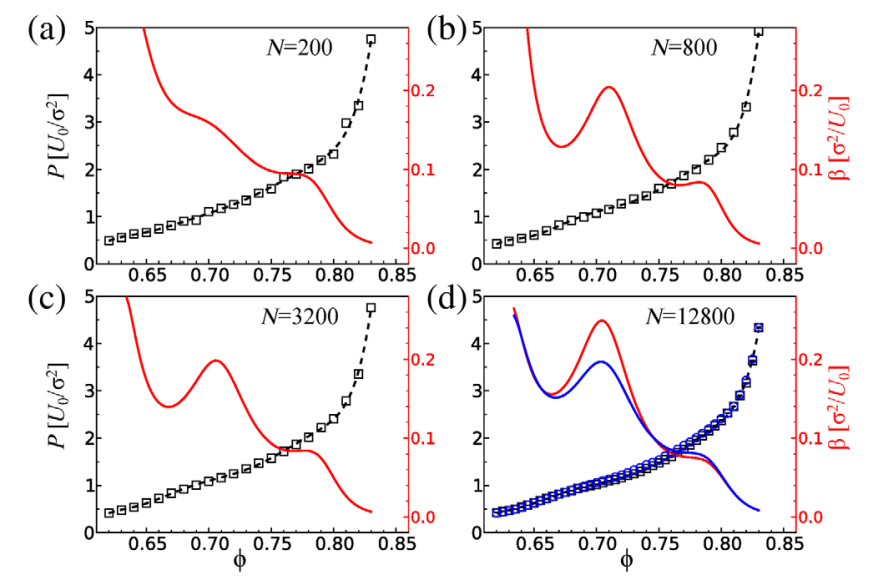

FIG. 12. $\operatorname{EOS} P(\phi)$ (squares) and compressibility $\beta$ (red curve) for the 2D binary systems with $N=200$ (a), 800 (b), 3200 (c), 12800 (d) particles. The reverse decompression process [blue curve in (d)] also shows the same five regimes as above and only a weak hysteresis around $\phi=0.7$.

(a)

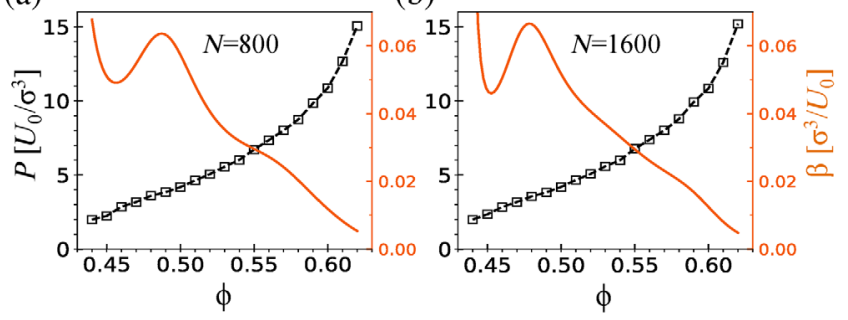

FIG. 13. EOS $P(\phi)$ (squares) and compressibility $\beta$ (red curve) for the 3D binary systems with $N=800$ (a) and 1600 (b) particles.

\section{APPENDIX E: SHEAR-INDUCED PLASTIC DEFORMATION}

We sheared the systems along the $x$ direction at a strain rate of $10^{-4} t_{0}{ }^{-1}$ with the Lees-Edwalds boundary condition along the $y$ direction. The stress increases linearly with strain at $<5 \%$ deformation, i.e., the elastic deformation regime, and plateaus at $>7 \%$ strain, i.e., the plastic flow regime [Fig. 4(a)]. Flow stress $\tau_{f}$, or the yield stress, was measured as the plateau height $[11,42]$ averaged over the strain range of $[0.2,0.5]$ in [Fig. 4(a)]. The linear elastic regime at small strains in Fig. 4(a) further demonstrates that the system is rigid under a shear, i.e., a solid. The system at a higher $\phi$ is more rigid, i.e., with a higher plateau height of the flow stress in Fig. 4(a), than the crystal at the low $\phi=0.64$; hence such a disordered and mechanically rigid system is a glass [1] rather than a liquid.

\section{APPENDIX F: DENSITY OF STATES}

We measured the vibrational modes and DOS by constructing the covariance matrix $[24,44,45,72]$ of particle displacements relative to their equilibrium positions, 


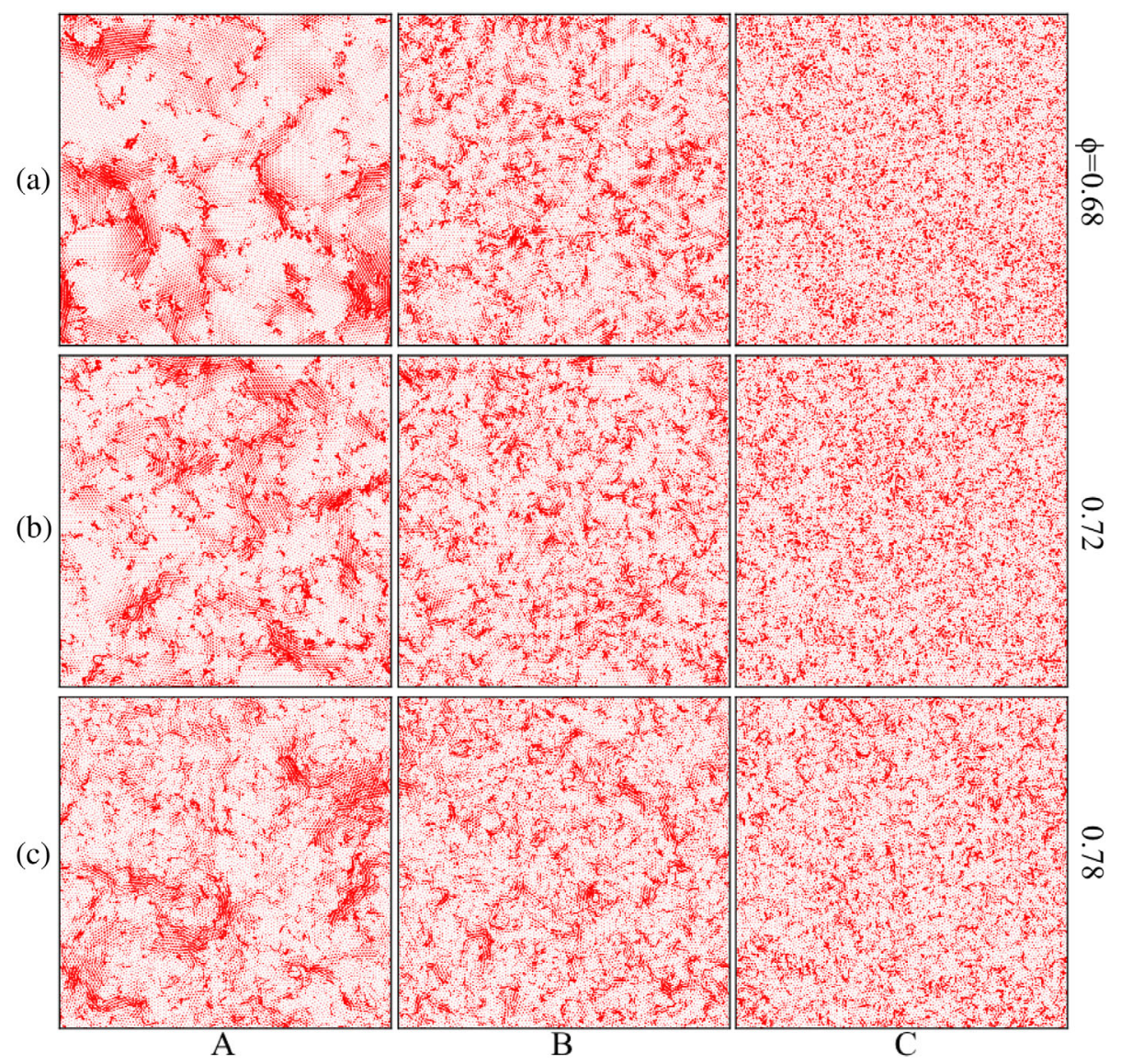

FIG. 14. Three vibrational normal modes with frequencies $\omega / \sqrt{U_{0} / m \sigma^{2}}=1.0$ (A), 5.5 (B), and 13.5 (C) for $\phi=0.68$ (a), 0.72 (b), and $0.78(\mathrm{c})$, respectively.

$$
C_{i j}=\left\langle\left(\mathbf{r}_{\mathbf{i}}(t)-\left\langle\mathbf{r}_{\mathbf{i}}(t)\right\rangle\right)\left(\mathbf{r}_{\mathbf{j}}(t)-\left\langle\mathbf{r}_{\mathbf{j}}(t)\right\rangle\right)\right\rangle,
$$

where $\mathbf{r}_{i}$ is particle $i$ 's position and \langle\rangle is the average over time. We diagonalized the matrix using LAPACKE, an interface of LAPACK [73] in the OPENBLAS library [74], and obtained the eigenvectors and eigenvalues $\lambda_{i}=U_{0} /\left(m \omega_{i}^{2}\right)$, where $\omega_{i}$ is the frequency of the $i$ th mode. Since our highest $\phi$ is still nowhere near the close packing $\phi_{\mathrm{cp}}$, the boson peak and lowfrequency plateau in typical glasses are not observable [75]. Figure 13 shows typical modes at low $\omega$. Low-frequency modes are more heterogeneous (column A in Fig. 14) with larger amplitudes at grain boundaries in the polycrystal regime and dispersed randomly in the glass regime.

[1] L. Berthier and G. Biroli, Theoretical Perspective on the Glass Transition and Amorphous Materials, Rev. Mod. Phys. 83, 587 (2011).

[2] Y. M. Wang, M. W. Chen, F. H. Zhou, and E. Ma, High Tensile Ductility in a Nanostructured Metal, Nature (London) 419, 912 (2002).

[3] B. Poudel, Q. Hao, Y. Ma, Y. C. Lan, A. Minnich, B. Yu, X. Yan, D. Z. Wang, A. Muto, D. Vashaee, X. Y. Chen, J. M. Liu, M.S. Dresselhaus, G. Chen, and Z.F. Ren,
High-Thermoelectric Performance of Nanostructured Bismuth Antimony Telluride Bulk Alloys, Science 320, 634 (2008).

[4] L. Lu, Y. F. Shen, X. H. Chen, L. H. Qian, and K. Lu, Ultrahigh Strength and High Electrical Conductivity in Copper, Science 304, 422 (2004).

[5] T. Chookajorn, H. A. Murdoch, and C. A. Schuh, Design of Stable Nanocrystalline Alloys, Science 337, 951 (2012).

[6] C. A. Schuh and A. C. Lund, Atomistic Basis for the Plastic Yield Criterion of Metallic Glass, Nat. Mater. 2, 449 (2003).

[7] E. Flenner and G. Szamel, Fundamental Differences between Glassy Dynamics in Two and Three Dimensions, Nat. Commun. 6, 7392 (2015).

[8] Y. H. Sun, A. Concustell, and A. L. Greer, Thermomechanical Processing of Metallic Glasses: Extending the Range of the Glassy State, Nat. Rev. Mater. 1, 16039 (2016).

[9] S. Vivek, C. P. Kelleher, P. M. Chaikin, and E. R. Weeks, Long-Wavelength Fluctuations and the Glass Transition in Two Dimensions and Three Dimensions, Proc. Natl. Acad. Sci. U.S.A. 114, 1850 (2017).

[10] B. Illing, S. Fritschi, H. Kaiser, C. L. Klix, G. Maret, and P. Keim, Mermin-Wagner Fluctuations in 2D Amorphous Solids, Proc. Natl. Acad. Sci. U.S.A. 114, 1856 (2017).

[11] J. Schiøtz and K. W. Jacobsen, A Maximum in the Strength of Nanocrystalline Copper, Science 301, 1357 (2003). 
[12] K. Lu, Stabilizing Nanostructures in Metals Using Grain and Twin Boundary Architectures, Nat. Rev. Mater. 1, 16019 (2016).

[13] J. Hu, Y. N. Shi, X. Sauvage, G. Sha, and K. Lu, Grain Boundary Stability Governs Hardening and Softening in Extremely Fine Nanograined Metals, Science 355, 1292 (2017).

[14] F. A. Lavergne, D. G. A. L. Aarts, and R. P. A. Dullens, Anomalous Grain Growth in a Polycrystalline Monolayer of Colloidal Hard Spheres, Phys. Rev. X 7, 041064 (2017).

[15] C. E. Cash, J. Wang, M. M. Martirossyan, B. K. Ludlow, A. E. Baptista, N. M. Brown, E. J. Weissler, J. Abacousnac, and S. J. Gerbode, Local Melting Attracts Grain Boundaries in Colloidal Polycrystals, Phys. Rev. Lett. 120, 018002 (2018).

[16] T. Kawasaki, T. Araki, and H. Tanaka, Correlation between Dynamic Heterogeneity and Medium-Range Order in TwoDimensional Glass-Forming Liquids, Phys. Rev. Lett. 99, 215701 (2007).

[17] T. Kawasaki and H. Tanaka, Formation of a Crystal Nucleus from Liquid, Proc. Natl. Acad. Sci. U.S.A. 107, 14036 (2010).

[18] H. Tanaka, T. Kawasaki, H. Shintani, and K. Watanabe, Critical-like Behaviour of Glass-Forming Liquids, Nat. Mater. 9, 324 (2010).

[19] E. Sanz, C. Valeriani, E. Zaccarelli, W. C. K. Poon, P. N. Pusey, and M. E. Cates, Crystallization Mechanism of Hard Sphere Glasses, Phys. Rev. Lett. 106, 215701 (2011).

[20] J. Ketkaew, Z. Liu, W. Chen, and J. Schroers, Critical Crystallization for Embrittlement in Metallic Glasses, Phys. Rev. Lett. 115, 265502 (2015).

[21] M. M. J. Treacy and K. B. Borisenko, The Local Structure of Amorphous Silicon, Science 335, 950 (2012).

[22] P. Yunker, Z. X. Zhang, and A. G. Yodh, Observation of the Disorder-Induced Crystal-to-Glass Transition, Phys. Rev. Lett. 104, 015701 (2010).

[23] C. P. Goodrich, A. J. Liu, and S. R. Nagel, Solids between the Mechanical Extremes of Order and Disorder, Nat. Phys. 10, 578 (2014).

[24] D. Kaya, N. L. Green, C. E. Maloney, and M. F. Islam, Normal Modes and Density of States of Disordered Colloidal Solids, Science 329, 656 (2010).

[25] H. Tong, P. Tan, and N. Xu, From Crystals to Disordered Crystals: A Hidden Order-Disorder Transition, Sci. Rep. 5, 15378 (2015).

[26] S. K. Deb, M. Wilding, M. Somayazulu, and P. F. McMillan, Pressure-Induced Amorphization and an AmorphousAmorphous Transition in Densified Porous Silicon, Nature (London) 414, 528 (2001).

[27] Y. C. Wang, W. Zhang, L. Y. Wang, Z. Zhuang, E. Ma, J. Li, and Z. W. Shan, In Situ TEM Study of Deformation-Induced Crystalline-to-Amorphous Transition in Silicon, NPG Asia Mater. 8, e291 (2016).

[28] O. Mishima, L. D. Calvert, and E. Whalley, "Melting Ice’ I at $77 \mathrm{~K}$ and 10 kbar: A New Method of Making Amorphous Solids, Nature (London) 310, 393 (1984).

[29] T. Egami and Y. Waseda, Atomic Size Effect on the Formability of Metallic Glasses, J. Non-Cryst. Solids 64, 113 (1984).
[30] O. V. Yazyev and Y. P. Chen, Polycrystalline Graphene and Other Two-Dimensional Materials, Nat. Nanotechnol. 9, 755 (2014).

[31] K. H. Nagamanasa, S. Gokhale, A. K. Sood, and R. Ganapathy, Direct Measurements of Growing Amorphous Order and Non-Monotonic Dynamic Correlations in a Colloidal Glass-Former, Nat. Phys. 11, 403 (2015).

[32] X. N. Yang, R. Liu, M. C. Yang, W. H. Wang, and K. Chen, Structures of Local Rearrangements in Soft Colloidal Glasses, Phys. Rev. Lett. 116, 238003 (2016).

[33] X. Cao, H. J. Zhang, and Y. L. Han, Release of Free-Volume Bubbles by Cooperative-Rearrangement Regions during the Deposition Growth of a Colloidal Glass, Nat. Commun. 8, 362 (2017).

[34] G. Malescio and G. Pellicane, Stripe Phases from Isotropic Repulsive Interactions, Nat. Mater. 2, 97 (2003).

[35] M. Sperl, E. Zaccarelli, F. Sciortino, P. Kumar, and H. E. Stanley, Disconnected Glass-Glass Transitions and Diffusion Anomalies in a Model with Two Repulsive Length Scales, Phys. Rev. Lett. 104, 145701 (2010).

[36] T. Dotera, T. Oshiro, and P. Ziherl, Mosaic Two-Lengthscale Quasicrystals, Nature (London) 506, 208 (2014).

[37] J. R. Trelewicz and C. A. Schuh, The Hall-Petch Breakdown in Nanocrystalline Metals: A Crossover to Glass-like Deformation, Acta Mater. 55, 5948 (2007).

[38] S. C. Kapfer and W. Krauth, Two-Dimensional Melting: From Liquid-Hexatic Coexistence to Continuous Transitions, Phys. Rev. Lett. 114, 035702 (2015).

[39] E. O. Hall, The Deformation and Ageing of Mild Steel: III Discussion of Results, Proc. Phys. Soc. London Sect. B 64, 747 (1951).

[40] N. J. Petch, The Cleavage Strength of Polycrystals, J. Iron Steel Inst. 174, 25 (1953).

[41] Z. C. Cordero, B. E. Knight, and C. A. Schuh, Six Decades of the Hall-Petch Effect-A Survey of Grain-Size Strengthening Studies on Pure Metals, Int. Mater. Rev. 61, 495 (2016).

[42] X. Y. Li, Y. J. Wei, L. Lu, K. Lu, and H. J. Gao, Dislocation Nucleation Governed Softening and Maximum Strength in Nano-Twinned Metals, Nature (London) 464, 877 (2010).

[43] Z. Wang, B. A. Sun, H. Y. Bai, and W. H. Wang, Evolution of Hidden Localized Flow during Glass-to-Liquid Transition in Metallic Glass, Nat. Commun. 5, 5823 (2014).

[44] A. Ghosh, V. K. Chikkadi, P. Schall, J. Kurchan, and D. Bonn, Density of States of Colloidal Glasses, Phys. Rev. Lett. 104, 248305 (2010).

[45] C. L. Klix, G. Maret, and P. Keim, Discontinuous Shear Modulus Determines the Glass Transition Temperature, Phys. Rev. X 5, 041033 (2015).

[46] Y. Kraftmakher, Equilibrium Vacancies and Thermophysical Properties of Metals, Phys. Rep. 299, 79 (1998).

[47] A. I. Chumakov et al., Role of Disorder in the Thermodynamics and Atomic Dynamics of Glasses, Phys. Rev. Lett. 112, 025502 (2014).

[48] J. M. Rieser, C. P. Goodrich, A. J. Liu, and D. J. Durian, Divergence of Voronoi Cell Anisotropy Vector: A ThresholdFree Characterization of Local Structure in Amorphous Materials, Phys. Rev. Lett. 116, 088001 (2016). 
[49] H. W. Sheng, H. Z. Liu, Y. Q. Cheng, J. Wen, P. L. Lee, W. K. Luo, S. D. Shastri, and E. Ma, Polyamorphism in a Metallic Glass, Nat. Mater. 6, 192 (2007).

[50] T. Voigtmann, Multiple Glasses in Asymmetric Binary Hard Spheres, Europhys. Lett. 96, 36006 (2011).

[51] C. Mayer, E. Zaccarelli, E. Stiakakis, C. N. Likos, F. Sciortino, A. Munam, M. Gauthier, N. Hadjichristidis, H. Iatrou, P. Tartaglia, H. Löwen, and D. Vlassopoulos, Asymmetric Caging in Soft Colloidal Mixtures, Nat. Mater. 7, 780 (2008).

[52] See Supplemental Material at http://link.aps.org/ supplemental/10.1103/PhysRevX.8.041023 for videos showing the structural and dynamical evolution at 0.68 (Video S1), 0.70 (Video S2), and 0.78 (Video S3).

[53] W. Mickel, S. C. Kapfer, G. E. Schröder-Turk, and K. Mecke, Shortcomings of the Bond Orientational Order Parameters for the Analysis of Disordered Particulate Matter, J. Chem. Phys. 138, 044501 (2013).

[54] B. Li, D. Zhou, and Y. L. Han, Assembly and Phase Transitions of Colloidal Crystals, Nat. Rev. Mater. 1, 15011 (2016).

[55] F. Turci, G. Tarjus, and C. P. Royall, From Glass Formation to Icosahedral Ordering by Curving Three-Dimensional Space, Phys. Rev. Lett. 118, 215501 (2017).

[56] K. J. Strandburg, Two-Dimensional Melting, Rev. Mod. Phys. 60, 161 (1988).

[57] E. P. Bernard and W. Krauth, Two-Step Melting in Two Dimensions: First-Order Liquid-Hexatic Transition, Phys. Rev. Lett. 107, 155704 (2011).

[58] P. Harrowell, Nonlinear Physics: Glass Transitions in Plane View, Nat. Phys. 2, 157 (2006).

[59] G. Tarjus, Glass Transitions May be Similar in Two and Three Dimensions, After All, Proc. Natl. Acad. Sci. U.S.A. 114, 2440 (2017).

[60] H. K. Mao, X. J. Chen, Y. Ding, B. Li, and L. Wang, Solids, Liquids, and Gases under High Pressure, Rev. Mod. Phys. 90, 015007 (2018).

[61] Q. S. Zeng, H. W. Sheng, Y. Ding, L. Wang, W. Yang, J. Z. Jiang, W. L. Mao, and H. K. Mao, Long-Range Topological Order in Metallic Glass, Science 332, 1404 (2011).
[62] M. N. Bannerman, R. Sargant, and L. Lue, DynamO: A Free General Event-Driven Molecular Dynamics Simulator, J. Comput. Chem. 32, 3329 (2011).

[63] F.H. Stillinger and B. D. Lubachevsky, CrystallineAmorphous Interface Packings for Disks and Spheres, J. Stat. Phys. 73, 497 (1993).

[64] W. K. Qi, A. P. Gantapara, and M. Dijkstra, Two-Stage Melting Induced by Dislocations and Grain Boundaries in Monolayers of Hard Spheres, Soft Matter 10, 5449 (2014).

[65] C. Rycroft, VORO++: A three-dimensional voronoi cell library in $\mathrm{C}++$, Chaos 19, 041111 (2009).

[66] Y. Peng, F. Wang, Z. R. Wang, A. M. Alsayed, Z. X. Zhang, A. G. Yodh, and Y. L. Han, Two-Step Nucleation Mechanism in Solid-Solid Phase Transitions, Nat. Mater. 14, 101 (2015).

[67] H. J. Zhang, S. M. Peng, X. S. Zhou, and X. Ju, Polymorphic Crystals Selected in the Nucleation Stage, Europhys. Lett. 107, 46002 (2014).

[68] P. R. ten Wolde, M. J. Ruiz-Montero, and D. Frenkel, Numerical Calculation of the Rate of Homogeneous GasLiquid Nucleation in a Lennard-Jones System, J. Chem. Phys. 104, 9932 (1996).

[69] Y. L. Han, N. Y. Ha, A. M. Alsayed, and A. G. Yodh, Melting of Two-Dimensional Tunable-Diameter Colloidal Crystals, Phys. Rev. E 77, 041406 (2008).

[70] D. Stauffer and A. Aharony, Introduction to Percolation Theory (Taylor \& Francis, Boca Raton, FL, 1994), Chap. 2.

[71] V. V. Vasisht, S. Saw, and S. Sastry, Liquid-liquid Critical Point in Supercooled Silicon, Nat. Phys. 7, 549 (2011).

[72] B. Li, F. Wang, D. Zhou, Y. Peng, R. Ni, and Y. L. Han, Modes of Surface Premelting in Colloidal Crystals Composed of Attractive Particles, Nature (London) 531, 485 (2016).

[73] E. Anderson, Z. J. Bai, C. Bischof, S. Blackford, J. Dongarra, J. Du Croz, A. Greenbaum, S. Hammarling, A. McKenney, and D. Sorensen, LAPACK Users' Guide (SIAM, Philadelphia, 1999), Vol. 9.

[74] X. Y. Zhang, Q. Wang, and C. Zaheer, openbLAS: An Optimized BLAS Library, http://xianyi.github.io/OpenBLAS.

[75] A. J. Liu and S. R. Nagel, The Jamming Transition and the Marginally Jammed Solid, Annu. Rev. Condens. Matter Phys. 1, 347 (2010). 